\title{
CONTINUOUS WAVELET TRANSFORM FOR THE DETECTION AND CLASSIFICATION OF EPILEPTIFORM ACTIVITY IN THE EEG
}

\author{
Hansjerg Goelz ${ }^{1,2^{*}}$, Richard D. Jones ${ }^{1,3}$, Philip J. Bones ${ }^{2}$ \\ ${ }^{1}$ Department of Medical Physics and Bioengineering, Christchurch Hospital, \\ ${ }^{2}$ Department of Electrical and Electronic Engineering, University of Canterbury, \\ ${ }^{3}$ Department of Medicine, Christchurch School of Medicine, University of Otago, \\ Christchurch, New Zealand. (*hangi@ieee.org)
}

\begin{abstract}
This paper outlines a novel approach to wavelet based detection of epileptiform activity in the EEG. A special complex-valued wavelet filter is used in a continuous wavelet transform (CWT). The response of wavelet coefficients (WCs) to epileptiform discharges (EDs) is measured with respect to artifact-free background activity (BG). A detector based on the WCs of a single scale could operate at $45 \%$ sensitivity without false alarms or at $99 \%$ sensitivity with 19 false alarms per minute on an artifact-free recording.

Key Words - continuous wavelet transform, spike detection, epileptiform discharge, epileptiform transient
\end{abstract}

\section{INTRODUCTION}

EDs appear as epileptiform transients (ETs) in one or more channels of the EEG. Efforts to automate the detection of EDs have made it clear that both spatial and temporal contextual information are crucial to the decision making process of EEGers [1]. Several approaches have been made to apply wavelet analysis to $\mathrm{ED}$ detection based on the discrete wavelet transform (DWT) [2] and CWT [3]. While there are advantages favouring the DWT (fast decomposition algorithm and inverse transform), detection based on the DWT can only fully respond to a target pattern if that pattern occurs at some dyadic translation coinciding with the temporal alignment of the DWT.

\section{METHODS}

Data: Data comprised 11 16-channel EEG recordings (bipolar, total $278 \mathrm{~min}$ ) containing 298 definite EDs (as determined by three independent EEGers) sampled at $200 \mathrm{~Hz}$. Wavelet Filter: Because optimal detection is achieved with a wavelet that correlates well with the target transients, we chose the 'psi' wavelet [4] for our analysis. This wavelet is complex-valued and has a real part which correlates strongly with the Mexican hat wavelet.

Evaluation: Each channel of the EEG was convolved with the wavelet filter ( 1 scale, centre frequency: $25 \mathrm{~Hz}$ ) to obtain the corresponding WCs. Local magnitude maxima were chosen for further analysis. At least two artifact-free 2000 $\mathrm{ms}$ periods were used to assess the distribution of the BG's $\log$ WCs. The mean $\log$ magnitude of the BG's WCs was subtracted to measure the strength of EDs with respect to a common reference. The channel showing the largest WC in response to an $\mathrm{ED}$ was chosen and two histograms were generated for $\log$ WCs of BG and EDs, respectively.

\section{RESULTS}

Figure 1 shows the histograms for BG and ED $\log$ WCs for a centre frequency of $25 \mathrm{~Hz}$. A reasonable separation of artifact-free background and definite EDs is achieved. A detector based on the WCs of this scale could operate at $45 \%$ sensitivity without false alarms, or at $99 \%$ sensitivity with 19 false alarms per minute. Similar distributions were obtained for two other scales (centre frequencies 12.5 and $50 \mathrm{~Hz}$, respectively).

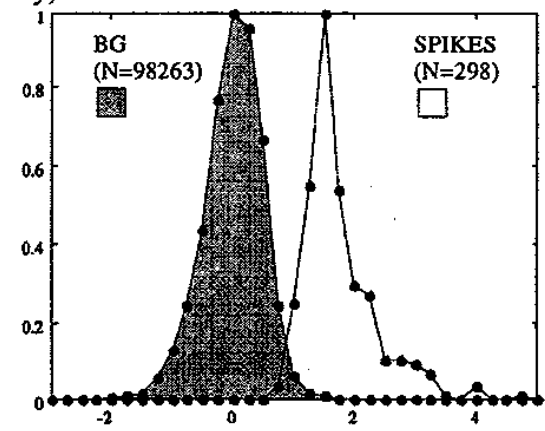

Figure 1: Distribution of $\log$ magnitude of wavelet coefficients for artifact-free background activity (BG) and definite epileptiform discharges (SPIKES).

\section{DISCUSSION}

The single scale detector outlined in this paper will be implemented as initial stage in a multistage detector. Classification of a detected transient will be based on concurrent exploration of three domains: time, scale and space.

\section{REFERENCES}

[1] A.A. Dingle, R.D. Jones, G.J. Carroll, and W.R. Fright, "A multistage system to detect epileptiform activity in the EEG," IEEE Trans. Biomed. Eng., vol. 40, 1260-1268, 1993.

[2] T. Kalayci and Ö. Özdamar, "Wavelet preprocessing for automated neural network detection of EEG spikes," IEEE Eng. Med. Biol., vol. 14, pp. 160-166, 1995.

[3] L. Senhadji, J.L. Dillenseger, F. Wendling, C. Rocha, and A. Kinie, "Wavelet analysis of EEG for three-dimensional mapping of epileptic events," Ann. Biomed. Eng., vol. 23, pp. 543-552, 1995.

[4] H. Gölz, F. Rösler, B. Röder, and E. Hennighausen, "Segregation of spatiotemporal components of auditory event related potentials," in Quantitative and Topological EEG and MEG analysis, H. Witte et al Eds. Jena: Druckhaus Meyer Verlag, pp. 238-241, 1997. 